\title{
Editorial
}

\section{Nature-Inspired Algorithms for Real-World Optimization Problems}

\author{
Wei Fang, ${ }^{1}$ Xiaodong Li, ${ }^{2}$ Mengjie Zhang, ${ }^{3}$ and Mengqi Hu${ }^{4}$ \\ ${ }^{1}$ School of IoT Engineering, Jiangnan University, No. 1800, Lihu Avenue, Wuxi 214122, China \\ ${ }^{2}$ School of Computer Science and IT, RMIT University, GPO Box 2476, Melbourne, VIC 3001, Australia \\ ${ }^{3}$ Evolutionary Computation Research Group, School of Engineering and Computer Science, Victoria University of Wellington, \\ P.O. Box 600, Wellington 6140, New Zealand \\ ${ }^{4}$ University of Illinois at Chicago, 842 W. Taylor Street, 2039 ERF, Chicago, IL 60661, USA
}

Correspondence should be addressed to Wei Fang; fangwei@jiangnan.edu.cn

Received 26 August 2015; Accepted 26 August 2015

Copyright (C) 2015 Wei Fang et al. This is an open access article distributed under the Creative Commons Attribution License, which permits unrestricted use, distribution, and reproduction in any medium, provided the original work is properly cited.

Nature-inspired algorithms are a set of novel problem-solving methodologies and approaches and have been attracting considerable attention for their good performance. Representative examples of nature-inspired algorithms include artificial neural networks (ANN), fuzzy systems (FS), evolutionary computing (EC), and swarm intelligence (SI), and they have been applied to solve many real-world problems. Despite the popularity of nature-inspired algorithms, many challenges remain which require further research efforts.

The contributions presented in this special issue include some latest developments of nature-inspired algorithms, such as genetic algorithm, particle swarm optimization, ant colony optimization, migrating birds optimization, neural networks, gravitational search algorithm, and their applications. Several real-world optimization problems have been studied by several nature-inspired algorithms.

K. G. Ing at al. present the application of gravitational search algorithm (GSA) in determining the optimal daily configuration of distribution network based on photovoltaic generation and system loading. The distribution network reconfiguration problem is formulated as a minimization problem to minimize the power loss of the distribution. Experimental results show that GSA with selection approach is a simple yet effective technique to minimize total daily power loss.

The work of E. Lalla-Ruiz et al. studies the improved migrating birds optimization (MBO) approach for solving two seaside problems, which are the Dynamic Berth Allocation Problem (DBAP) and Quay Crane Scheduling Problem (QCSP). MBO approach can solve these two problems with high-quality solutions with a small short computational cost, which makes this technique a competitive method for frequently seaside operations either performed individually or embedded into real decision-support systems.

The paper by I. G. Hidalgo et al. integrates genetic algorithm (GA) with Strength Pareto Evolutionary Algorithm (SPEA) and ant colony optimization (ACO) to deal with the short-term scheduling problem. The problem is solved by the proposed two hybrid approaches in two phases. The experimental results on two hydroelectric plants show that both approaches produce good performance for the optimal dynamic dispatch in the short-term operation of hydroelectric plants.

S. Demirel et al. focus on the optimal design of ultrawideband (UWB) low-noise amplifier (LNA) based on the support vector regression machine (SVRM) microstrip line model. Particle swarm optimization (PSO) algorithm has been employed in the solving procedure for two parameters resulting in good performance in terms of accuracy and fast convergence.

F. Kamaruzaman et al. propose the coincidence detection (CD) classifier with two learning methods based on the Spiking Neural Network (SNN). The proposed method can produce an output spike pattern from an input pair identical 
to the discrete Spike Response Model (SRM) with significantly lower floating operations and a much faster processing time.

The papers included in this special issue are of high quality, hopefully making useful contributions to the research area of nature-inspired algorithms.

\section{Acknowledgments}

Dr. Wei Fang acknowledges the support from the National Nature Science Foundation of China (Grants nos. 61105128, 61170119, and 61373055), the Nature Science Foundation of Jiangsu Province, China (Grants nos. BK20131106, BK20130161, and BK20130160), the Postdoctoral Science Foundation of China (Grant no. 2014M560390), the Fundamental Research Funds for the Central Universities, China (Grant no. JUSRP51410B), and Six Talent Peaks' Project of Jiangsu Province (Grant no. DZXX-025). As guest editors, we would like to thank the contributing authors and the reviewers for their hard work in preparing and reviewing the submissions.

Wei Fang Xiaodong Li Mengjie Zhang Mengqi $\mathrm{Hu}$ 


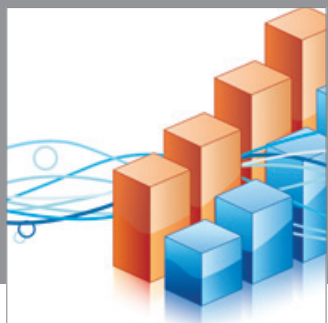

Advances in

Operations Research

mansans

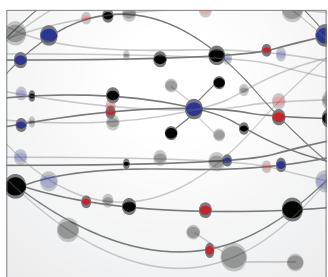

The Scientific World Journal
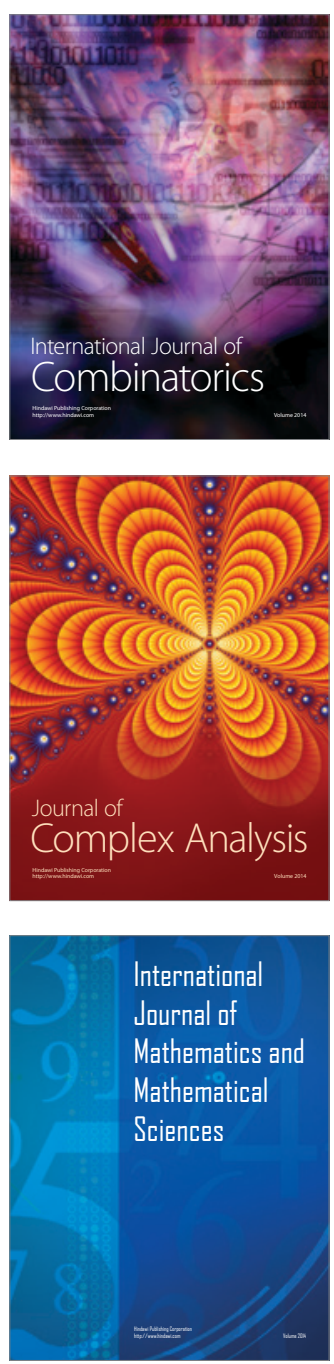
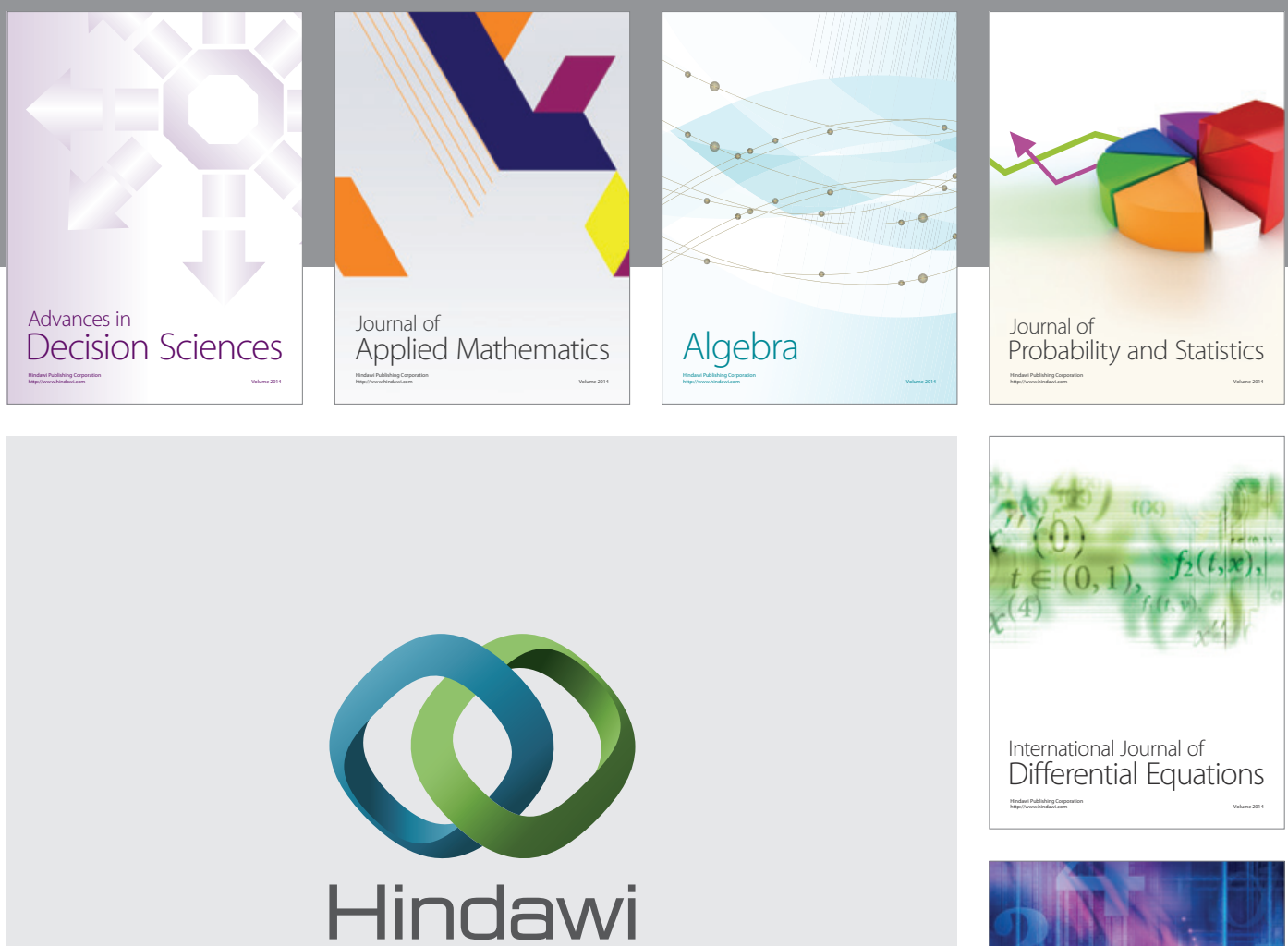

Submit your manuscripts at http://www.hindawi.com
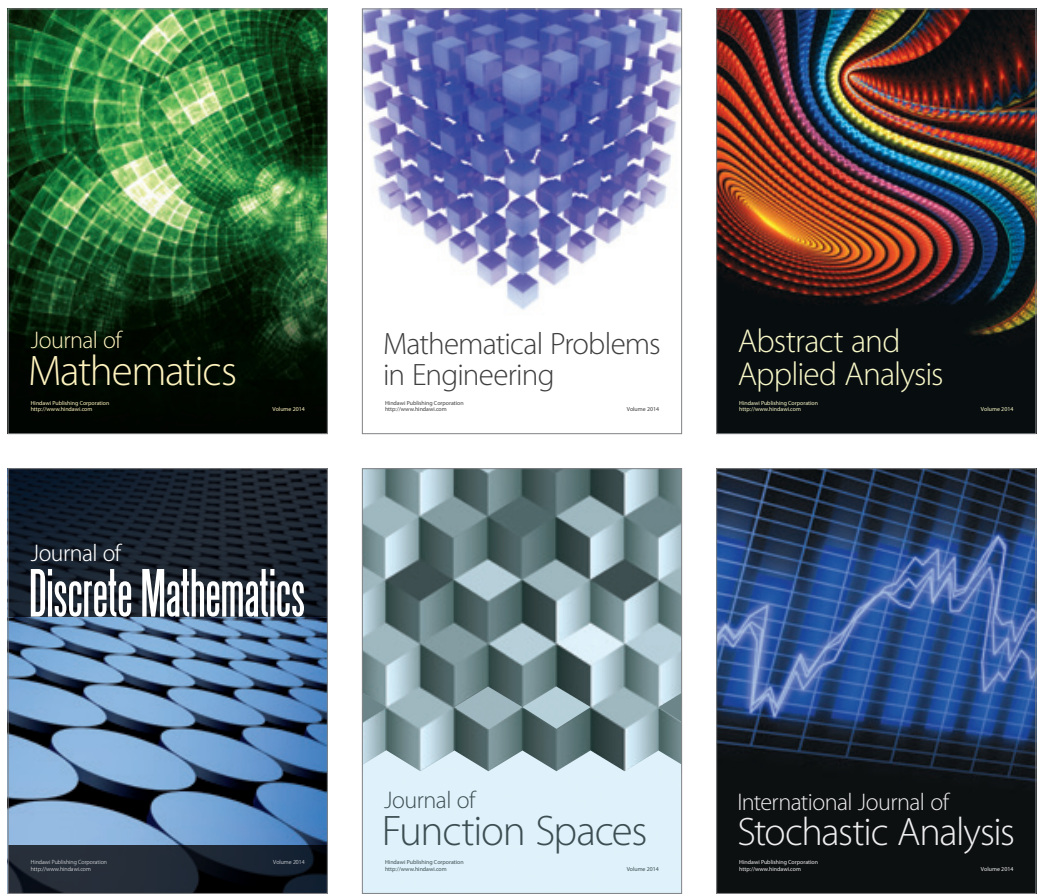

Journal of

Function Spaces

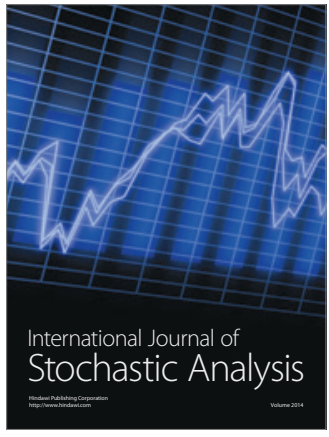

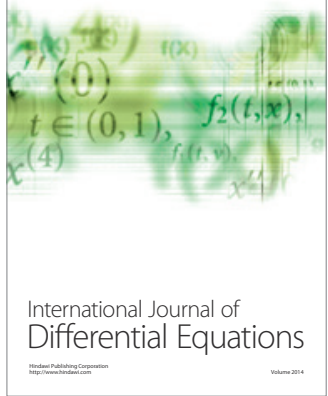
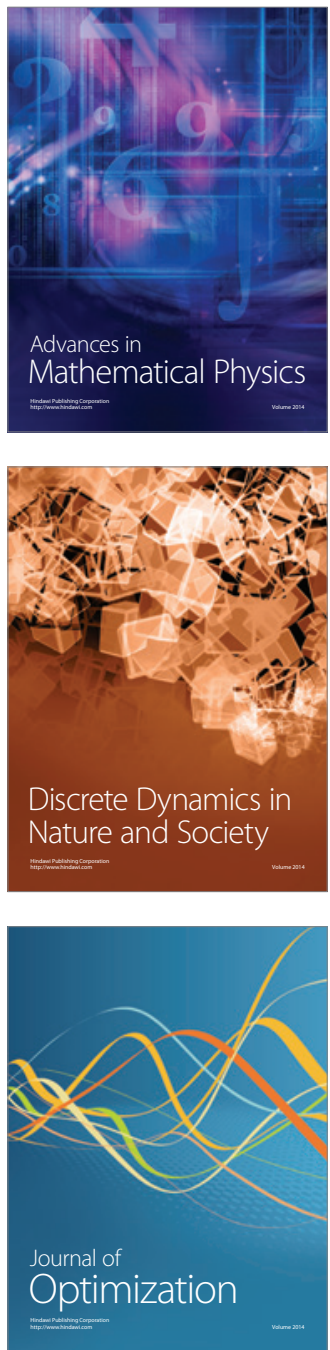